Supporting Information

\title{
Fast Automated Approach for the Derivation of Acellular Extracellular Matrix Scaffolds from Porcine Soft Tissues
}

Andreea Badileanu\#, Camilo Mora-Navarro", Ana M. Gracioso Martins, Mario E. Garcia, Daphne Sze, Emily W. Ozpinar, Lewis Gaffney, Jeffrey R. Enders, Ryan C. Branski, and Donald O. Freytes*

List of abbreviations $\mathbf{S 1}$

Method S1

Figures S1-S5

Table S1 


\section{List of abbreviations S1}

\begin{tabular}{l|l}
\hline Abbreviation & Explanation \\
ECM & Extracellular Matrix \\
Col & Type I Collagen hydrogel \\
TCP & Tissue Culture Plastic \\
sH-ECM & sheet Heart-Extracellular Matrix \\
sH-ECMh & sheet Heart-Extracellular Matrix hydrogel \\
mH-ECM & micronized Heart-Extracellular Matrix \\
mH-ECM-O & micronized Heart-Extracellular Matrix Optimized \\
mH-ECMh-O & micronized Heart-Extracellular Matrix hydrogel Optimized \\
sVFLP-ECM & sheet Vocal Fold Lamina Propria-Extracellular Matrix \\
sVFLP-ECMh & $\begin{array}{l}\text { sheet Vocal Fold Lamina Propria-Extracellular Matrix hydrogel } \\
\text { micronized Vocal Fold Lamina Propria-Extracellular Matrix }\end{array}$ \\
mVFLP-ECM & $\begin{array}{l}\text { micronized Vocal Fold Lamina Propria-Extracellular Matrix } \\
\text { hydrogel }\end{array}$ \\
mVFLP-ECMh
\end{tabular}

\section{Method S1 Gelation kinetics}

After hydrogel preparation (Section 2.5) $100 \mu \mathrm{L}$ of hydrogel/well at least in triplicate were aliquoted in a 96-well plate and kept on ice until measurement. The samples were measured using a BioTek SYNERGYneo2 multimode reader pre-heated to $37^{\circ} \mathrm{C}$. Absorbance measurements were taken at $405 \mathrm{~nm}$ every 1 minute for 90 minutes. The data was normalized using the following equation:

$$
\text { Normalized absorbance }_{405 \mathrm{~nm}}=\frac{\left(A_{x}-A_{\min }\right)}{\left(A_{\max }-A_{\min }\right)}
$$

Where $A_{x}=$ experimental measurement, $A_{\min }=$ minimum absorbance, $A_{\max }=$ maximum absorbance. The data was graphed using Prism 8.0. 
Figure S1 Final decellularized product placed inside the inline filtration bioreactor.

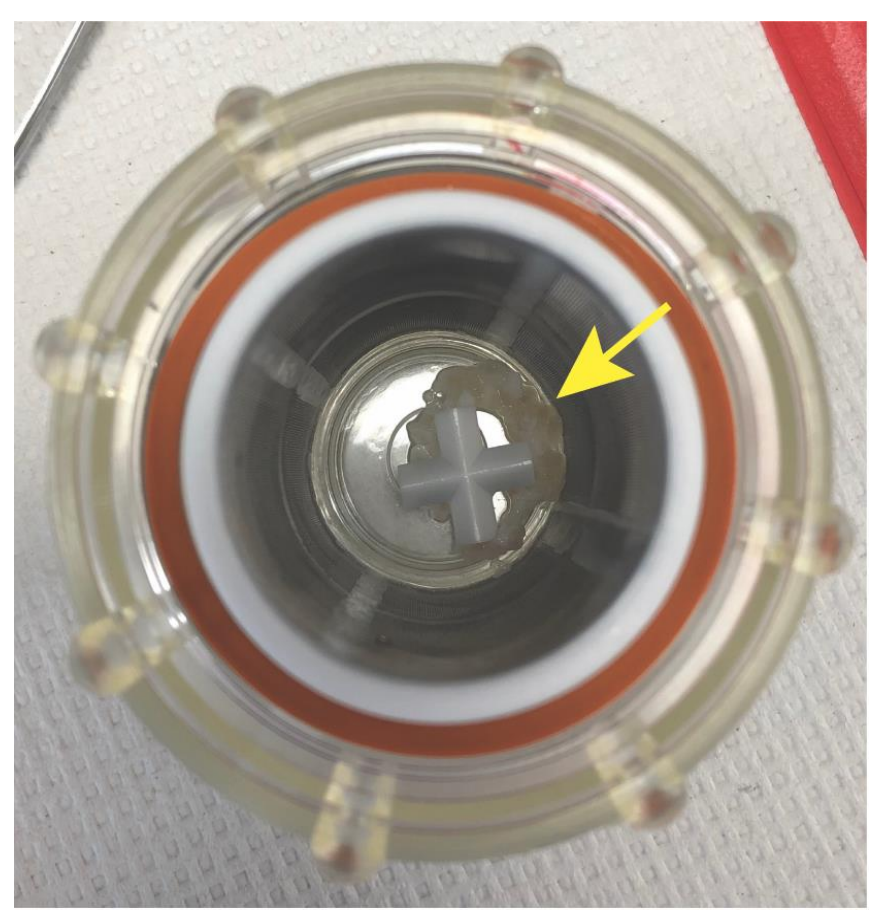


Figure S2 Histological analysis showing Hematoxylin and Eosin (H\&E) stain. Box and Whisker plot showing double stranded DNA (dsDNA) quantification per mg of dry tissue for native, wet micronized (W), and freeze-dried (D) decellularized sH$\mathrm{ECM}$ and $\mathrm{mH}-\mathrm{ECM}$ after 3 and/or 9 hours.

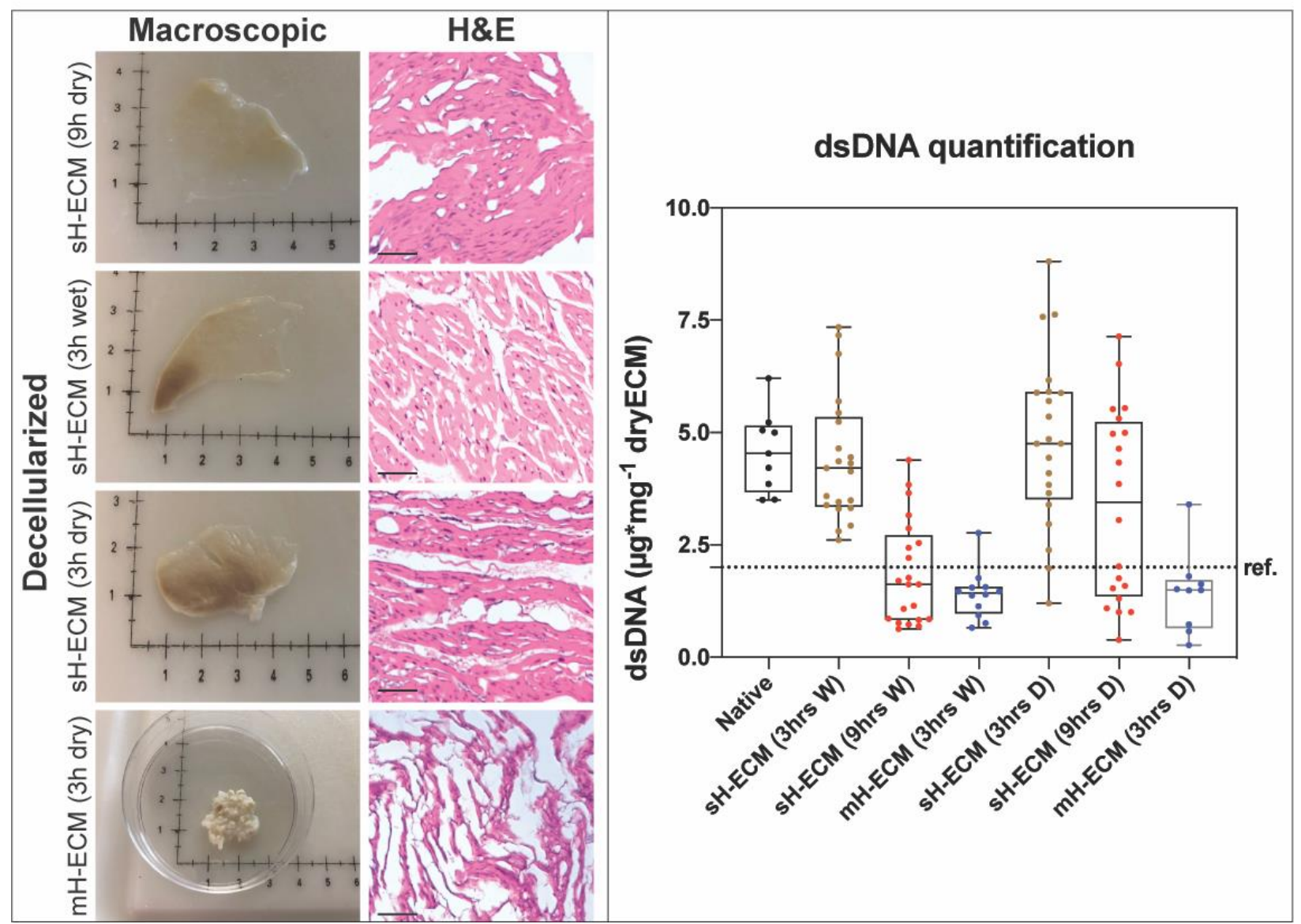

Three independent organs were decellularized at least three independent times $(n=9)$. The error bars represent the standard error of the mean (SEM). ref. $\sim 2 \mu \mathrm{g} / \mathrm{mg}$ (for Urinary Bladder Matrix-UBM). W \& $D$ were the pre-treatment conditions for the tissue to be decellularized $\mathrm{W}=$ wet micronized, $\mathrm{D}=$ freeze-dried. Scale bar $=360 \mu \mathrm{m}$. 
Figure S3 Gene Ontology (GO)

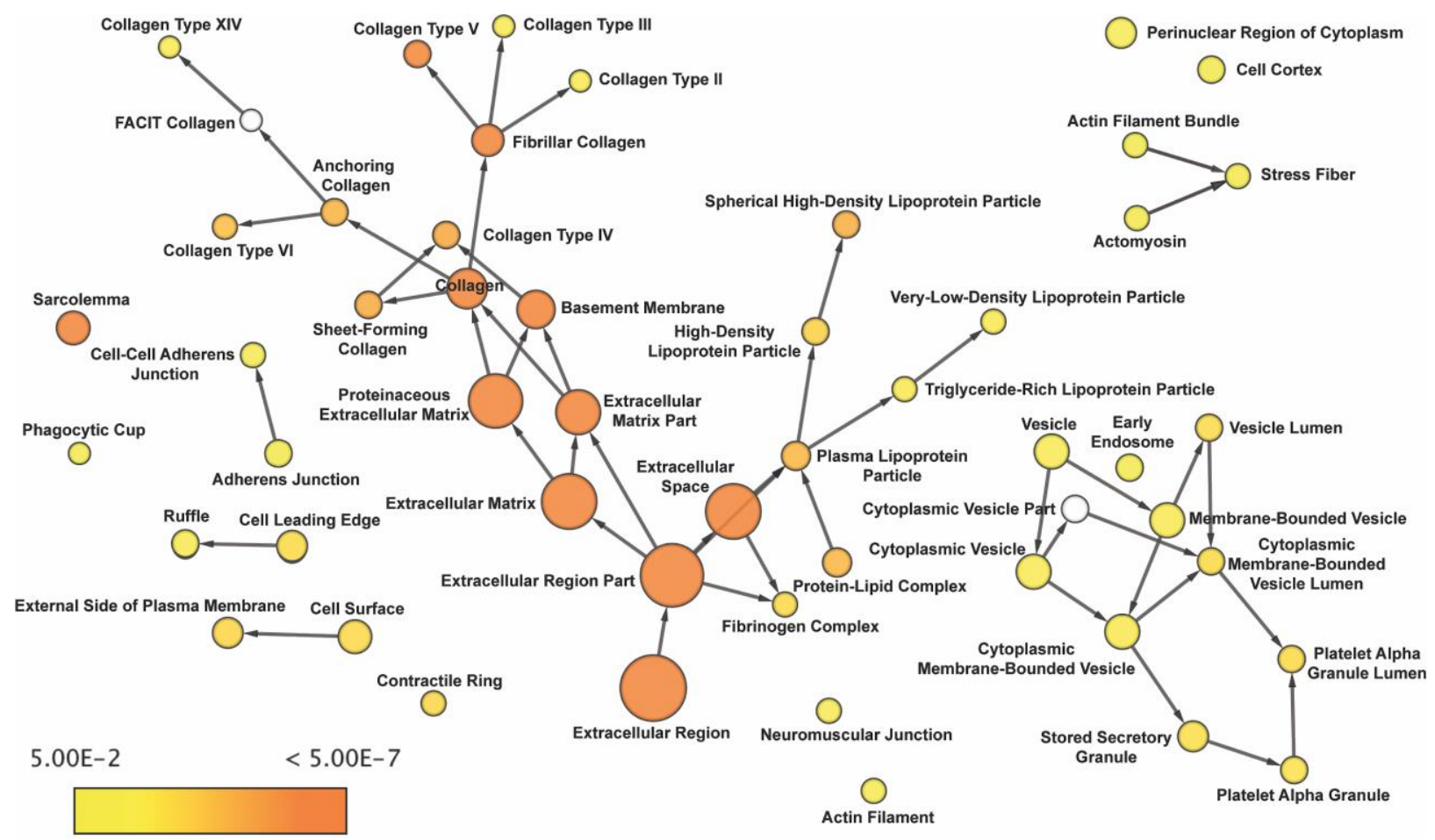

Gene ontology (GO) based on cellular components using the proteins in Figure $5 \mathrm{C}$ and extracted from Proteome Discoverer 2.4.0.305 as input in Cystoscope V3.0 and the application Bingo to plot overrepresenting analysis. The scale bar represents the $\mathrm{P}$-value for the nodes. 
Figure S4 Gelation kinetics curves

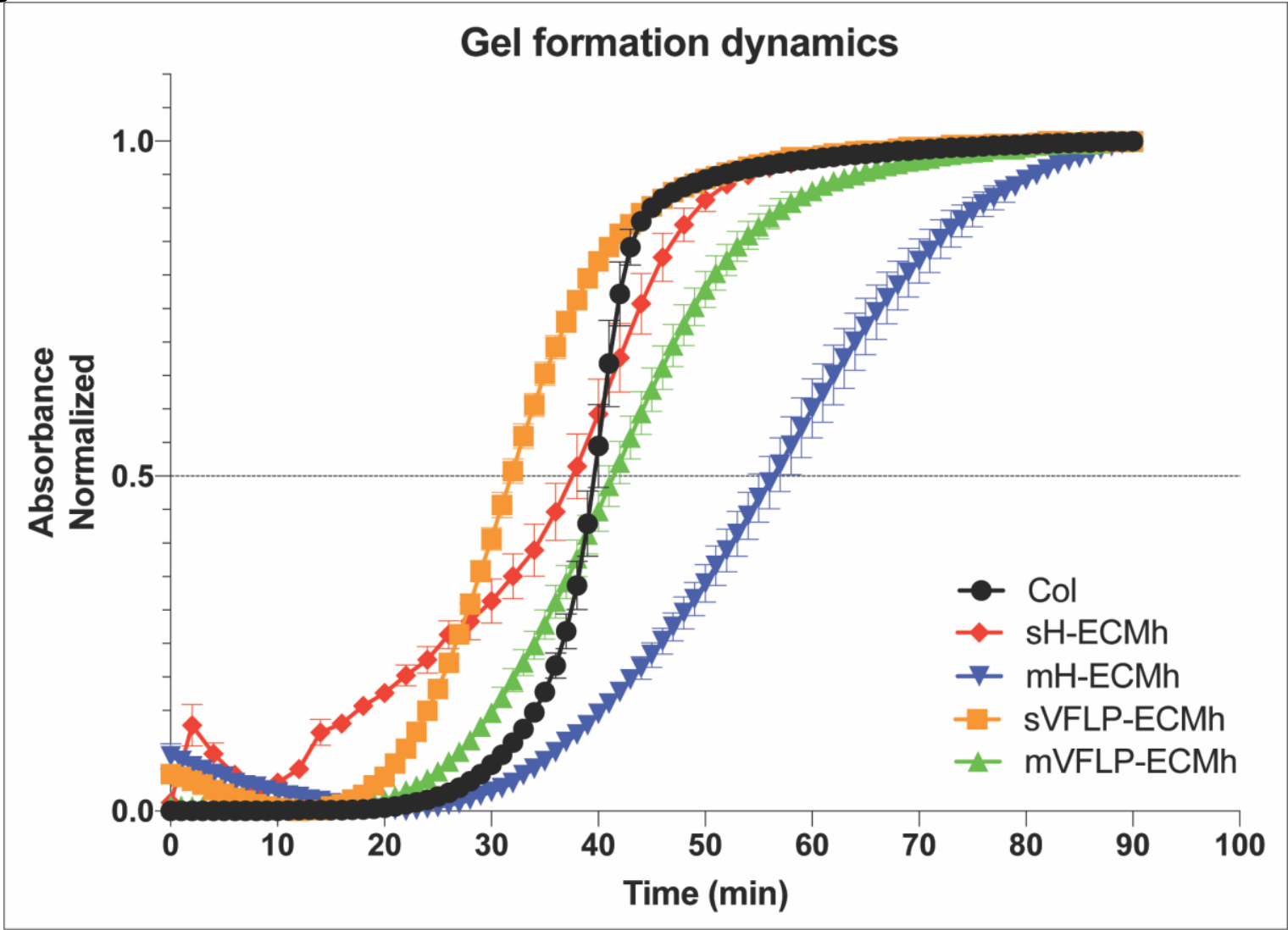

The curves were plotted based on the normalized absorbance at $405 \mathrm{~nm}$ for sH-ECMh, $\mathrm{mH}$-ECMh, sVFLP-ECMh, mVFLP-ECMh, and collagen type I hydrogel (Col) control. The error bars represent the SEM of at least three replicates. 
Figure S5 Differences in selected cellular proteins between automated ( $\mathrm{mH}-\mathrm{ECM}$ ) vs. manual (sH-ECM).

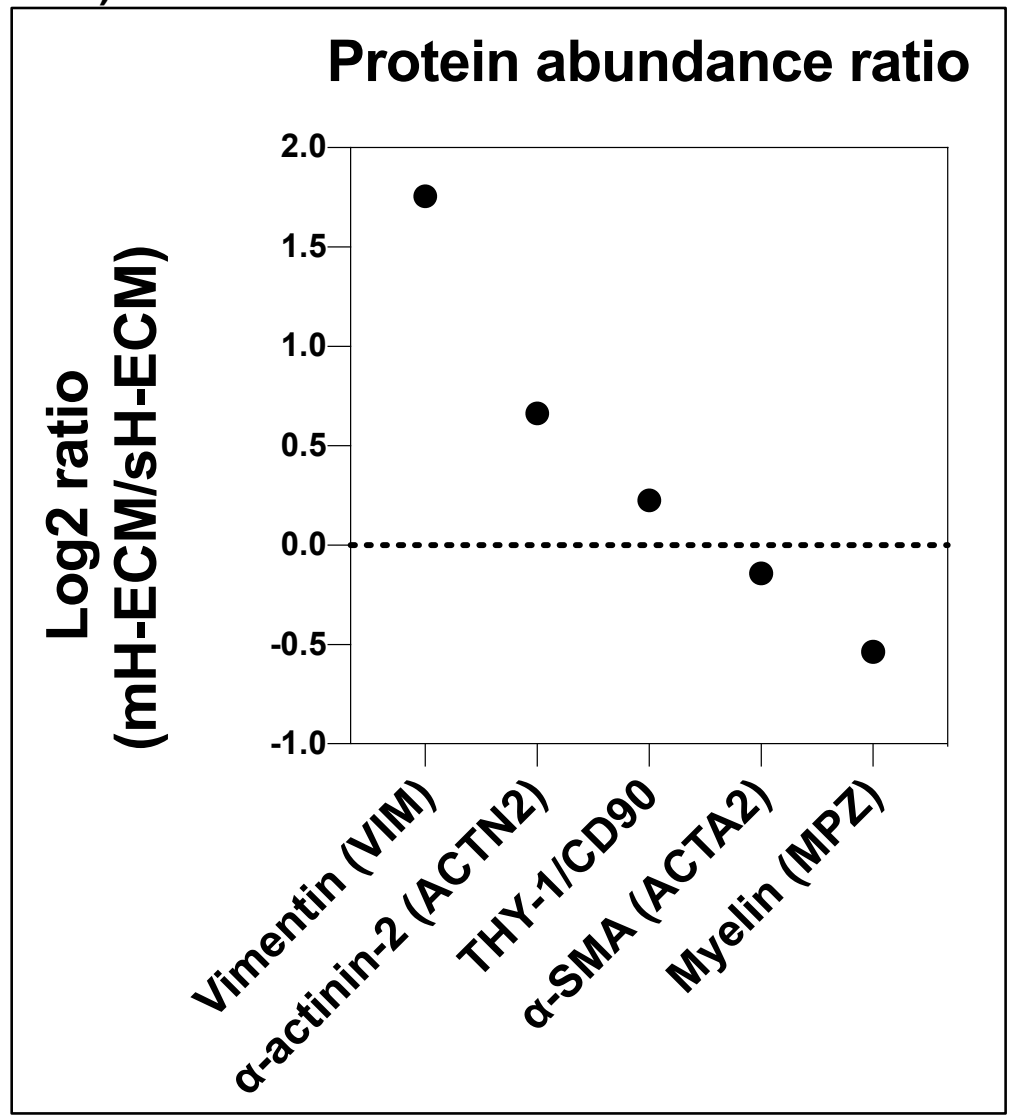

A Log2 ratio equal to $0(\cdots \cdots)$ indicates the 3 -hour method $(\mathrm{mH}-\mathrm{ECM})$ yielded the same abundance for the protein than the 9-hour method ( $\mathrm{sH}-\mathrm{ECM}$ ). 
Table S1 Approximate total number of manual steps calculated based on steps involving tissue preparation and manual reagent changes.

\begin{tabular}{|c|c|c|c|}
\hline \multirow[b]{2}{*}{ Decellularization Protocol } & \multicolumn{2}{|c|}{$\begin{array}{c}\text { Approx. Number of Manual } \\
\text { Steps }\end{array}$} & \multirow{2}{*}{$\begin{array}{c}\text { Total Number } \\
\text { of Manual } \\
\text { Steps }\end{array}$} \\
\hline & $\begin{array}{l}\text { Tissue } \\
\text { Preparation }\end{array}$ & $\begin{array}{l}\text { Manual } \\
\text { Reagent } \\
\text { Changes }\end{array}$ & \\
\hline $\mathrm{mH}-\mathrm{ECM}^{*}$ & 4 & 1 & 5 \\
\hline mVFLP-ECM* & 6 & 1 & 7 \\
\hline Sheet-Heart (Freytes et al.) & 3 & 15 & 18 \\
\hline Sheet-Heart (Singelyn et al.) & 2 & 4 & 6 \\
\hline Sheet-Heart (Shah et al.) & 2 & 19 & 21 \\
\hline Sheet-VFLP (Wrona et al.) & 3 & 9 & 12 \\
\hline Sheet-VFLP (Tse et al.) & 3 & 5 & 8 \\
\hline Sheet-VFLP (Xu et al.) & 2 & 5 & 7 \\
\hline Sheet-UBM (Freytes et al.) & 3 & 5 & 8 \\
\hline Sheet-Aorta (Pellegata et al.) & 6 & 1 & 7 \\
\hline Whole-Lungs (Price et al.) & 4 & 1 & 5 \\
\hline Whole-Kidneys (Poornejad et al.) & 4 & 1 & 5 \\
\hline Whole-Heart (Momtahan et al.) & 4 & 1 & 5 \\
\hline
\end{tabular}

